Scientific Visualization, 2021, volume 13, number 3, pages 47 - 57, DOI: 10.26583/sv.13.3.05

\title{
High-Speed Shadowgraphy of the Interaction of an Oblique Shock Wave in a Channel with a Surface Sliding Discharge
}

\author{
I.V. Mursenkova ${ }^{1}$, Yu. Liao ${ }^{2}$, P.Yu. Ulanov3, L. Shi4 \\ Lomonosov Moscow State University, Russia, 119991, Moscow, 119991, Leninskie Gory, 1 \\ ${ }^{1}$ ORCID: 0ooo-0002-7181-4533, murs i@physics.msu.ru \\ 2 ORCID: 0000-0002-5776-1949,565164346@qq.com \\ 3 ORCID: 0000-0002-8465-0240, ulapaveloo9@gmail.com \\ 4 ORCID: 0000-0002-1212-3804, shilino010@gmail.com
}

\begin{abstract}
The spatial structure of a supersonic flow with an oblique shock wave is studied experimentally during nanosecond surface sliding discharge initiation in a discharge chamber of a shock tube. An inclined shock wave was generated in a supersonic flow around a small obstacle on the lower wall of the channel. A surface sliding discharge was initiated on the upper wall of the discharge chamber directly in the region of interaction of the inclined shock wave with the boundary layer. High-speed shadowgraphy visualization of the flow field was carried out with a frequency of up to 150,000 frames per second at Mach numbers of flows up to 1.70. The discharge characteristics was recorded at different stages of flow and showed dependence on the type of interaction of the oblique shock wave with the boundary layer. It is established that the shock wave dynamics from the discharge depends on the discharge mode and the flow Mach number. According to the digital shadowgraph image processing, the shock waves generated by the discharge affects the shock-wave structure of the flow during $100 \mu \mathrm{s}$.
\end{abstract}

Keywords: high-speed shadowgraphy, supersonic flow, oblique shock wave, boundary layer, nanosecond surface discharge, digital image processing.

\section{Introduction}

Low-temperature plasma has been studied because of its significant potential for high speed flow control [1-6] and for plasma assisted combustion [7, 8] in aerospace science. The effects of different types of electrical discharges on airflow and streamlined bodies are discussed widely during the last three decades [1-4]. The main advantage of this control is the small actuation time, which makes actuator effective in a wide frequency range for gasdynamic flows control from stationary flows to turbulent and separated flows. Actuators are used to reduce surface friction, to influence the laminar-turbulent transition, to the position of separation zones and shock waves near the streamlined surface [2-6], to control the ignition of fuels and combustion $[4,7]$.

When a pulse of energy is inserted into a gas by an electric discharge, a rapid change in the state of the gas occurs in the region of the discharge current leads to the formation and movement of shock waves, pressure waves and rarefaction waves $[2-4,6,8]$. The main part of the electrical energy of the discharges in the air goes to the excitation of vibrational and electronic degrees of freedom of molecules [9]. A rather large proportion of electrical energy goes into translational degrees of freedom of gas molecules in the microsecond time interval [2-4]. The other part transforms into thermal energy in the process of vibrationaltranslational relaxation within several milliseconds [9]. The ion motion plays a key role in the plasma dynamics in the boundary layer and the spatial distribution of input energy release, as discussed in experimental and modeling studies [1, 2, 4]. Some investigations 
focused on the shock wave generation in supersonic flow and the plasma control of shock wave-boundary layer interaction $[2,3,5,6]$. Dynamics of discharges have not been studied in detail at operation in a high-speed flow.

For the effective action on high-speed gas flows, it is necessary to study the development of discharges in air flows, determine the mechanism of their interaction with shock waves. The study of plasma formations in a high-speed flow makes it possible to optimally solve technological problems and problems in the field of plasma gas dynamics. Moreover it is necessary to determine the structure of the supersonic flow after discharge. In high-speed flows in the channels, the formation of separation zones is possible when the boundary layer interacts with an oblique shock wave [10, 11], and the search for methods for controlling such zones remains relevant [12, 13], including the study of physical processes in the plasma of nanosecond discharges discharge in an inhomogeneous flow $[14,15]$.

Nanosecond distributed surface sliding discharge (plasma sheet) is a system of channels sliding over dielectric surface $[3,6,13]$. The discharge enables to realize energy input in gas layer of $\sim 0.5 \mathrm{~mm}$ near dielectric surface $[3,5]$ and can be used as an actuator for the control of a near-surface flow [3, 6, 13]. The near-surface flow influences the discharge channels $[3,6]$. The interaction of vortex zone and the relaxing plasma can lead to inhomogeneous discharge current $[14,15]$. This study is focused on the structure of a supersonic flow in a shock tube channel with inclined shock wave and on the details of surface sliding discharge dynamics in a supersonic airflow, which is important for understanding of the discharge physics and processes of plasma-flow interaction. The experimental visualization of the flow was realized based on high-speed shadowgraphy. The dynamic of shock-wave structure of a supersonic flow when flowing around a small obstacle was determined as well as the boundary layer on the wall and the region of interaction of the boundary layer with an oblique shock wave. The structure of the flow was analyzed after the initiation of a pulsed surface sliding discharge in the region of intersection of an inclined shock wave and a boundary layer.

\section{Experimental approach}

\subsection{Experimental setup}

The experiments were carried out on a shock tube with a rectangular channel of $24 \times 48$ $\mathrm{mm}^{2}[3,5]$. Supersonic air flows with a speed of $660-1370 \mathrm{~m} / \mathrm{s}$ were generated behind plane shock waves with Mach numbers $M=2.5-5.2$. The flow Mach numbers reached $\mathrm{M}_{1}=1.7 \mathrm{O}$, the Reynolds numbers of the flows were $\sim 10^{5}$ at a density of $0.01-0.15 \mathrm{~kg} / \mathrm{m}^{3}$. The thickness of the laminar boundary layer on the channel walls did not exceed $1 \mathrm{~mm}$ [5]. Two side walls of the test chamber of the shock tube were plane-parallel quartz glasses 170 mm long, which made it possible to carry out optical diagnostics (Fig. 1). On the lower and upper walls of the discharge chamber, electrodes of surface sliding discharges $100 \mathrm{~mm}$ long with an inter-electrode distance of $30 \mathrm{~mm}$ were located. A synchronizing system from signals of piezoelectric pressure sensors in the shock tube channel made it possible to initiate a discharge at a given time of gas dynamic process. In the experiments, a surface sliding discharge of nanosecond duration was initiated on the upper wall of the discharge chamber $80-800 \mu$ s after the initial shock wave passed the obstacle. The development of the discharge in the region of interaction of an inclined shock wave with the boundary layer and the gas-dynamic flow field after the discharge were investigated. Photo registration was carried out and 9-frame registration of the discharge radiation was carried out with a Ko11 BIFO ICCD camera. Emission spectra and the discharge current were recorded. High-speed shadowgraphy was used to study the plane shock wave diffraction on obstacle and the flow pattern evolution prior to and after the pulse surface sliding discharge. 


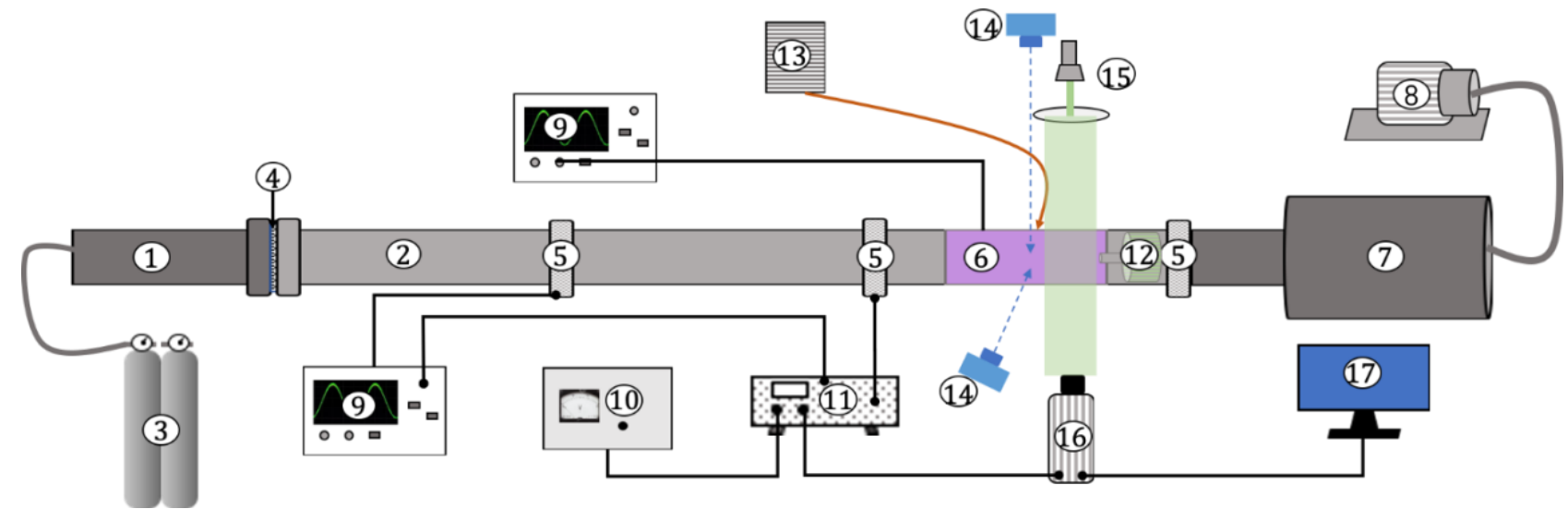

Figure 1. Experimental setup: 1 - driver section, 2 - driven section, 3 - helium, 4 diaphragm section, 5 - pressure sensors, 6 - discharge chamber, 7 - dump tank, 8 - vacuum pump, 9 - oscilloscopes, 10 - discharge triggering unit, 11 - delay generator, 12 - shunt, 13 spectrometer, 14 - photo cameras, 15 - shadowgraphy optics, 16 - high-speed video camera, 17 - PC.

\subsection{Direct shadowgraphy system}

The optical scheme of the direct shadowgraphy consists of a laser light source, diverging and collimating lenses, and a prism (Fig. 2). The lenses formed a plane-parallel light beam $\sim 40 \mathrm{~mm}$ in diameter. The prism was placed so that the beam passed through the discharge chamber perpendicular to the quartz glasses and hit the CCD matrix of the high-speed video camera. The light source was a continuous wave laser $(532 \mathrm{~nm})$. Shadowgraph images of the gas-dynamic flow field were recorded with a high-speed video camera with a frame rate of 150,000 frames per second. The frame exposure was $1 \mu \mathrm{s}$, the image size on the matrix was $256 \times 144$ pixels. Experimental shadowgraph images were processed with background subtraction. Then the images were processed with the intensity scanning program in different directions to obtain quantitative information about the motion of shock-wave structures.

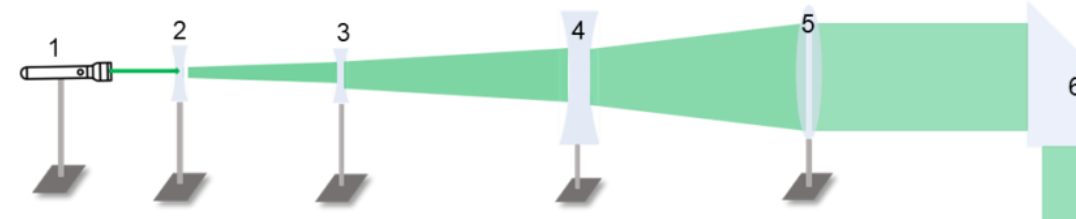

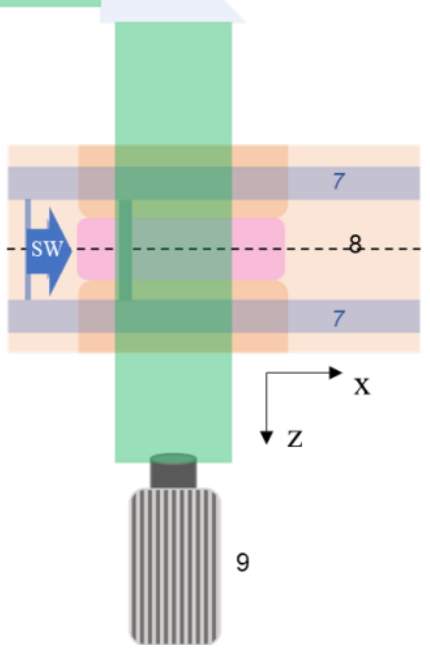

Figure 2. Direct shadowgraphy arrangement: 1 - laser; 2-4 - diverging lenses; 5 collimating lens; 6 - prism; 7 - quartz glasses; 8 - discharge chamber; 9 - high-speed video camera. 


\subsection{The supersonic flow in the channel around obstacle}

A dielectric obstacle in the form of a parallelepiped with dimensions of $48,0 \times 6,2 \times 1,9$ $\mathrm{mm}^{3}$ is located on the lower wall of the discharge chamber at a distance of $30 \mathrm{~mm}$ from the beginning of the electrodes (Fig. 3, a). The long part of the obstacle was perpendicular to the walls of the chamber. High-speed shadowgraphy of the flow field in the discharge chamber was carried out at all stages of its development from the beginning of diffraction of the initial plane shock wave on the obstacle to the end of a homogeneous co-current flow behind the initial shock wave.

Quasi-stationary flow around the obstacle was formed after the diffraction of the initial plane shock wave by the obstacle. Figure $3 \mathrm{~b}$ shows a sequence of images of the flow field in a channel with total length of about $80 \mathrm{~mm}$ (channel height is $24 \mathrm{~mm}$ ). Each image is composed of shadowgraph images from three separate experiments. The high repeatability of the processes under the same initial conditions makes it possible to reconstruct the flow field. Two upper images show the initial stages of diffraction of a plane shock wave, corresponding to the moments of time up to $20 \mu$ s from the moment the shock wave touches the leading edge of the obstacle. The upper part of the initial plane shock wave moves to the right, and the diffracted part of shock front moves to the left of the obstacle. At the unsteady stage of the flow up to $200 \mu \mathrm{s}$, an oblique shock wave forms behind the bottom of the obstacle and disturbances in front of the obstacle (images 4-6). The images 7-9 corresponds to the quasi-stationary stage of the flow with the steady-state positions of the shock waves in the channel. In the last image, the shock-wave configuration is modified and moves to the left after the end of the uniform co-current flow and the arrival of rarefaction waves. Boundary layers are formed on the channel walls in the flow behind a plane shock wave. First, the boundary layer is laminar, then it becomes turbulent at a certain distance from the shock front [5].

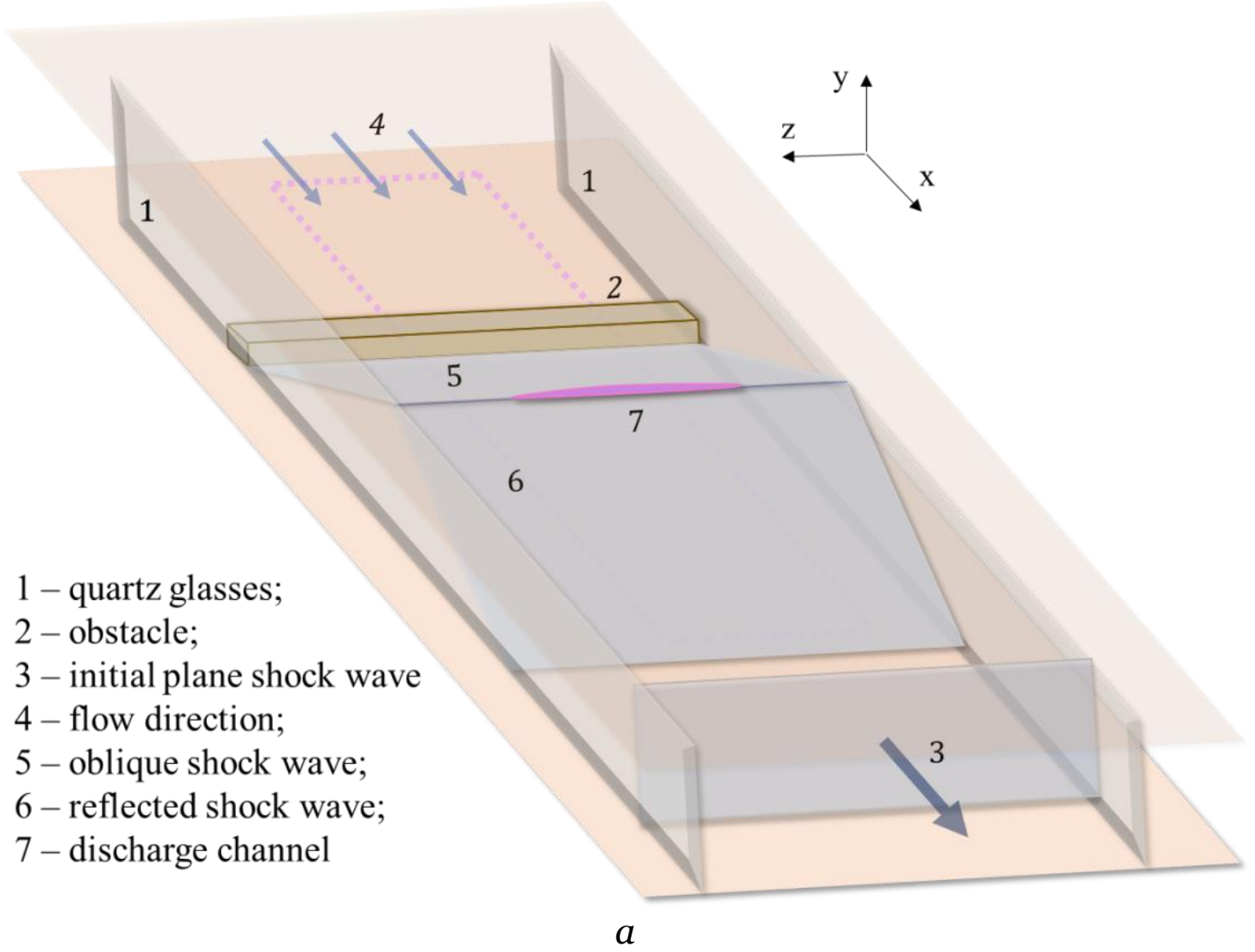



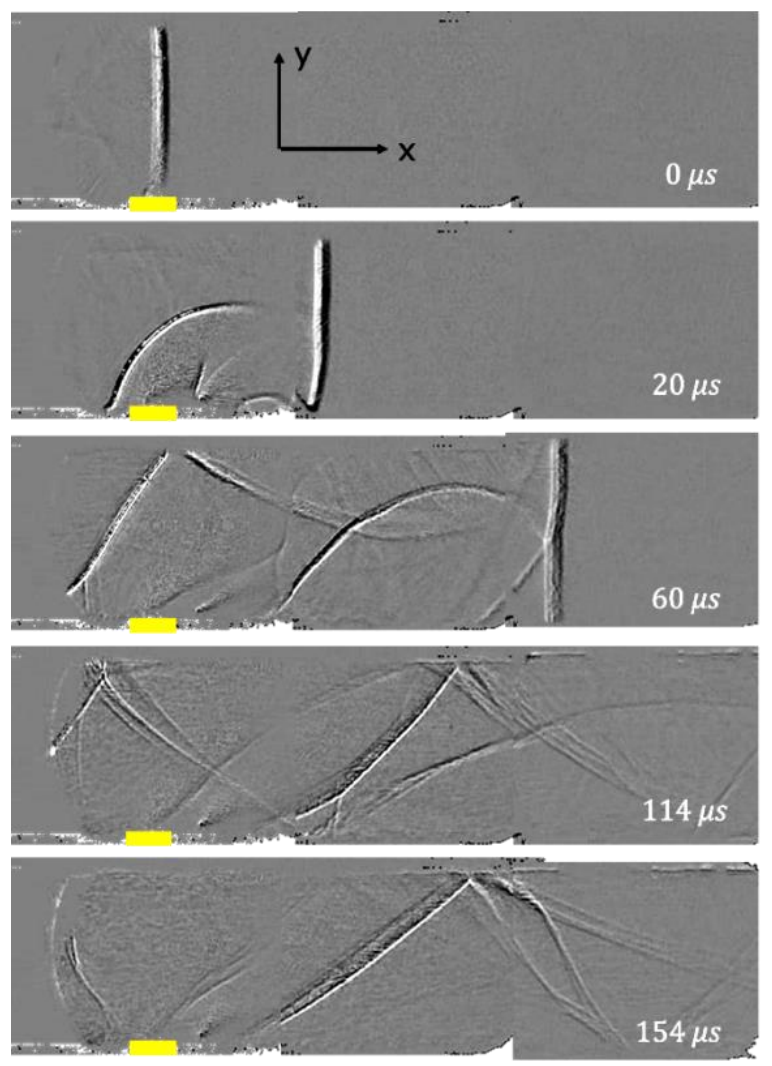

Figure 3. (a) Schematic of a steady shock-wave configuration; (b) The sequence of shadowgraph images of flow field after the interaction of plane shock wave with obstacle. Shadowgraph images are taken for initial shock wave number 3.50, initial pressure 25 torr. The zero-time corresponds to the moment when the initial plane shock wave contacts the obstacle (yellow rectangular on the lower wall).

Analysis of high-speed imaging showed that the formation of a quasi-stationary flow occurs within 90-150 $\mu$ s after the plane shock wave passes the obstacle. Its duration is about 150-500 $\mu \mathrm{s}$, which corresponds to numerical simulation [15]. The total duration of a homogeneous co-current flow behind a plane shock wave is 180-650 $\mu$ s depending on the Mach number of the initial shock wave (Fig. 4 a). An inclined shock wave forms behind the obstacle and interacts with the boundary layer on the upper wall of the discharge chamber. The angle of inclination of the shock wave is from 35 to 43 degrees in experiments depending on the flow Mach number. Reflection of an inclined shock wave from the boundary layer depends on the Mach number of the flow $\mathrm{M}_{1}$ and the boundary layer, as seen in the shadow images. Figure $4 \mathrm{~b}$ shows the increased near-wall flow regions of interaction of a laminar and turbulent boundary layer with an oblique shock wave of $5 \times 12$ $\mathrm{mm}^{2}$ in size. It is seen that the thickness of the laminar boundary layer is less than $0.5 \mathrm{~mm}$, and the thickness of the turbulent boundary layer does not exceed $1 \mathrm{~mm}$. Interaction with the boundary layer can be with separation of the flow or without separation [6-8]. In both cases, a region of low density is formed [15]. 


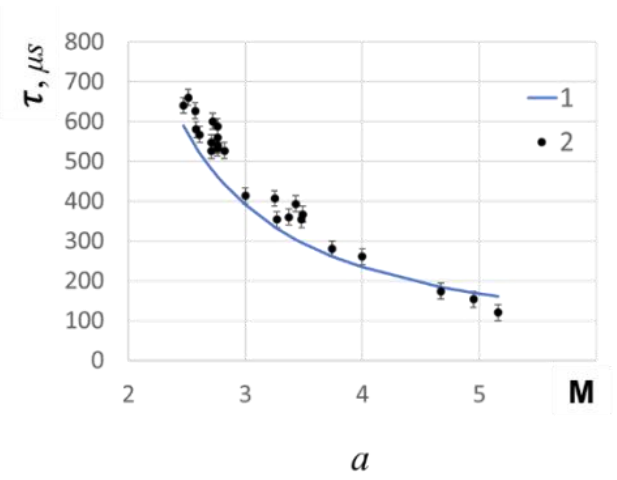

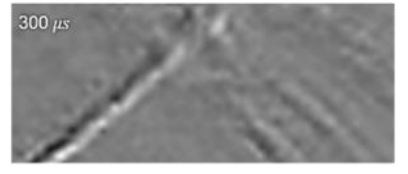

$M_{1}=1.28$

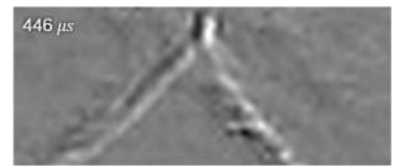

$M_{1}=1.51$

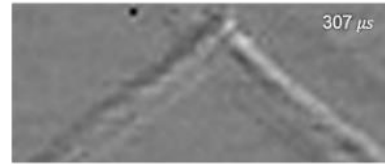

$b$

Figure 4. a) Duration of a homogeneous co-current flow in the shock tube depending on the Mach number of shock wave: 1 - theory [16], 2 - experiment; b) shadowgraph images of the oblique shock wave interacting with a laminar boundary layer (top) and turbulent boundary layer (bottom). The zero-time corresponds to the moment when the initial plane shock wave contacts the obstacle.

\subsection{Surface sliding discharge characteristics in supersonic flow}

A surface sliding discharge was initiated in experiments at a pulse voltage of $22-25 \mathrm{kV}$. The discharge current was $\sim 1 \mathrm{kA}$, the current duration was less than $500 \mathrm{~ns}$. The main energy input to the gas occurs within $150 \mathrm{~ns}$, i.e. almost instantaneously compared to the characteristic gas-dynamic time. Significant energy input into a thin gas layer leads to the formation of shock waves from the channels [3]. The short duration of the discharge ( $100 \mathrm{~ns}$ ) and the small thickness of the plasma layer, comparable to the thickness of the boundary layer in the shock tube $(\sim 1 \mathrm{~mm})$, make it possible to act almost instantly on the near-wall flow in a supersonic flow.

The development of a pulsed discharge is determined by the local value of the reduced electric field $E / N$ ( $E$ is the electric field strength, $N$ is the concentration of molecules), on which the plasma conductivity and discharge current depend [9]. Therefore, in an inhomogeneous air flow with an oblique shock wave, a surface sliding discharge develops as a single channel located in a region of low density formed during the interaction of an oblique shock wave with the boundary layer (Fig. 5 c, d), [15]. In experimental photo images, the discharge channel looks like an intensively emitting band about $10 \mathrm{~mm}$ wide. The image in Fig. 5 d obviously shows the clear boundaries of the discharge channel. The emission spectrum of the discharge in the flow is characterized by a high intensity of the continuum, indicating a high concentration of electrons [15].
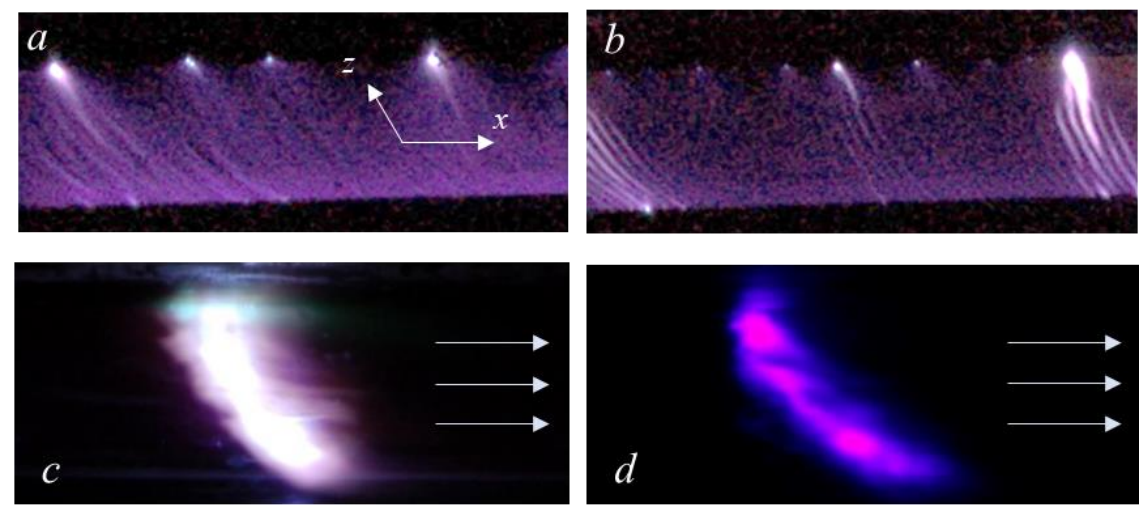

Figure 5. Photo images of a surface sliding discharge in motionless air at $0.05 \mathrm{\kappa г} / \mathrm{M}^{3}$ (a) and at $0.25 \mathrm{\kappa г} / \mathrm{M}^{3}$ density (b) and of discharge in supersonic air flow with inclined shock wave (c, d) at flow Mach number 1.27 and $0.15 \mathrm{~kg} / \mathrm{m}^{3}$ density. The image (d) was registered through an optical filter that transmits radiation with a wavelength of $405 \mathrm{~nm}$.

Arrows indicate the flow direction. 
The duration of the discharge glow was determined on the basis of registration with a 9-frame ICCD camera. Fig. 6 a, b shows photo images and 9-frame images of the discharge in motionless air and in a flow with an oblique shock wave. The glow time of the discharge channel in the flow is more than $4 \mu \mathrm{s}$. Fig. $6 \mathrm{c}$ shows the time dependences of the glow intensity and the current waveform. After the end of the discharge current, the afterglow of the current channel is observed with a decay time of $\sim 2 \mu \mathrm{s}$.
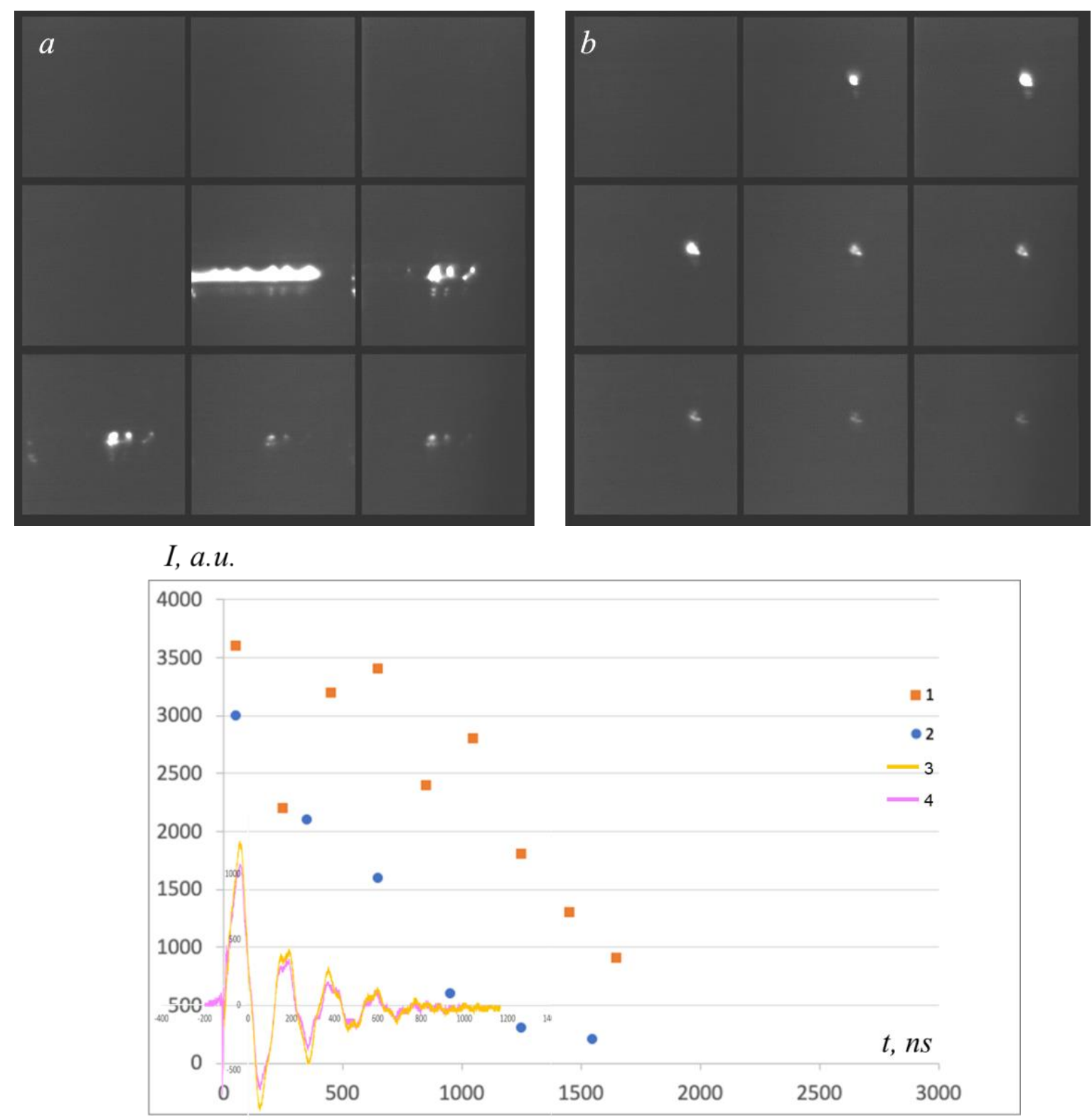

c

Figure 6. ICCD images of surface sliding discharge in motionless air at $0.085 \mathrm{~kg} / \mathrm{m}^{3}$ density (a) and in supersonic flow with inclined shock wave (b) at flow Mach number 1.27 and $0.15 \mathrm{~kg} / \mathrm{m}^{3}$ density. ICCD images were taken with exposure times of $100 \mathrm{~ns}$. Time intervals between frames were $100 \mathrm{~ns}$ (a) and $500 \mathrm{~ns}$ (b). Time dependences of discharge radiation intensity (c) in air flow (1) and in motionless air (2), discharge current waveforms in air flow (3) and in motionless air (4).

\section{The dynamics of supersonic flow after initiation of a surface sliding discharge}

Shadowgraphy imaging of the flow field after the discharge shows a shock wave propagation in the flow from the discharge channel, the front shape of which is close to 
semi-cylindrical at the initial stage of motion. Shock wave moves downward, away from the channel, and shifts to the right in the direction of the flow, rapidly damping (Fig. 7 a). Different velocities of the wave front parts lead to a change in the shape of the front over time. The movement of the shock wave significantly changes the structure of the flow in the discharge chamber. A heat trace is formed from the discharge region, which propagates in the boundary layer. It has been experimentally established that the discharge regime as well as shock wave dynamics depend on the Mach number of the flow and the type of interaction of the oblique shock wave with the boundary layer.

Shadowgraphy images were processed to obtain information on horizontal and vertical motion of the shock wave generated by the discharge channel. To determine the vertical displacement, the vertical coordinate of the shock front point located at the greatest distance from the upper edge of the image was taken. Figure $7 \mathrm{~b}$ shows the result of processing high-speed images obtained in different experiments of discharge initiation in air flows with Mach numbers of 1.27. It is seen that the dynamics of the vertical motion of the generated shock wave has good repeatability. The vertical velocity of the shock front during the first $7 \mu \mathrm{s}$ is about $1100 \mathrm{~m} / \mathrm{s}$, then falls to $700 \mathrm{~m} / \mathrm{s}$. The intensity of the shock wave depends on the magnitude of the current and the geometry of the region of discharge current, which, in turn, depends on the type of interaction of the oblique shock wave with the boundary layer. Within $\sim 100 \mu \mathrm{s}$, the flow pattern in the channel changes; then the quasi-stationary shock-wave flow configuration with an oblique shock wave is restored.
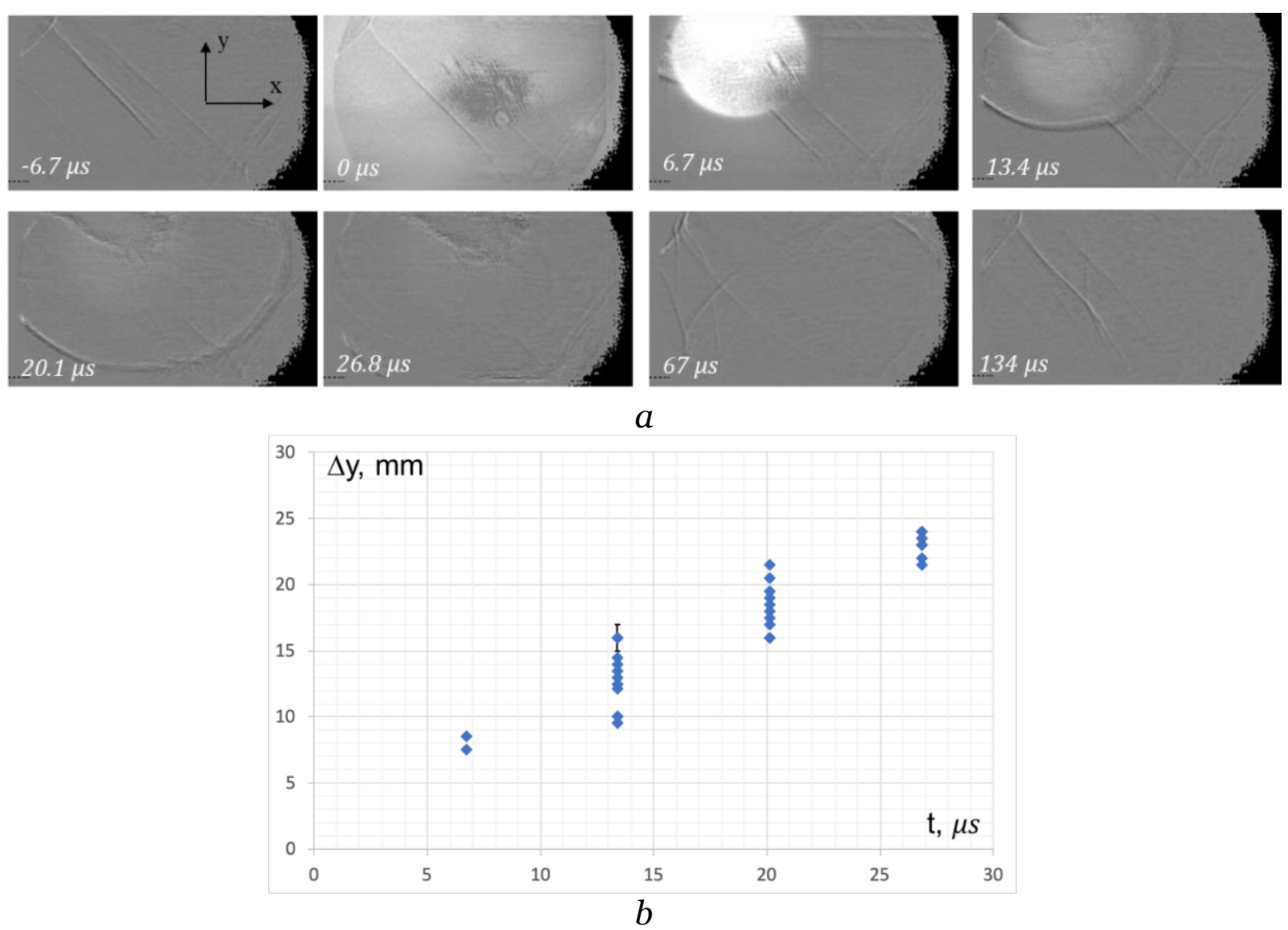

Figure 7. The sequence of shadowgraph images of flow field after the discharge (a) and the time dependence of vertical displacement of shock wave from discharge channel (b). Shadowgraph images are taken for flow Mach number 1.27 and $0.15 \mathrm{~kg} / \mathrm{m}^{3}$ density. The zero-time corresponds to the moment of discharge. The flow is from left to right. 


\section{Conclusion}

The spatial structure of a supersonic flow with an oblique shock wave upon initiation of a nanosecond surface sliding discharge in the discharge chamber of a shock tube is studied experimentally using high-speed shadowgraphy at a frequency of up to 150,000 frames per second. Investigation of the flow field was carried out in supersonic flows at Mach numbers of 1.20-1.68. Digital processing of sequences of obtained shadowgraph images showed that the shock-wave structure of the flow remains disturbed for more than $100 \mu \mathrm{s}$. Thus, supersonic flow with an oblique shock wave in the channel is affected by the movement of the shock waves generated by the discharge. The shock wave dynamics depends on the Mach number of the flow and the discharge parameters. The implementation of such an effect on the flow, taking into account the development of a surface sliding discharge in high-speed flows, can be used to control flows. Altogether, the obtained result can serve as a base for simulating the real time disturbed flow associated with movement of the shock wave generated by the discharge channel and studying downstream events.

\section{Acknowledgments}

The work was supported by RFBR grant 19-08-00661.

\section{References}

1. Bayoda D., Benard N., Moreau E. Nanosecond pulsed sliding dielectric barrier discharge plasma actuator for airflow control: Electrical, optical, and mechanical characteristics. // J. Appl. Phys., 2015. V. 118. 063301. https://doi.org/10.1063/1.4927844

2. Leonov S.B., Adamovich I.V., and Soloviev V.R. Dynamics of near-surface electric discharges and mechanisms of their interaction with the airflow. // Plasma Sources Sci. Technol., 2016. 25 063001. https://doi.org/10.1088/0963-0252/25/6/063001

3. Mursenkova I.V., Znamenskaya I.A., Lutsky A.E. Influence of shock waves from plasma actuators on transonic and supersonic airflow. // J. Phys. D: Appl. Phys. 2018. V. 51. N 5. 105201. https://doi.org/10.1088/1361-6463/aaa838

4. Starikovskiy A.Yu. and Aleksandrov N.L. Gasdynamic Flow control by ultrafast local heating in a strongly nonequilibrium pulsed pPlasma. // Plasma Physics Reports, 2021. V. 47. P. 148-209. https://doi.org/10.1134/S1063780X21020069

5. Znamenskaya I.A., Latfullin D.F. and Mursenkova I.V. Laminar-turbulent transition in a supersonic boundary layer during initiation of a pulsed surface discharge. // Technical $\begin{array}{lllllll}\text { Physics } & \text { Letters. } & 2008 . & \text { V. } 34 . & \text { No. } & \text { 8. } & \text { 668-670. }\end{array}$ https://doi.org/10.1134/S1063785008080130

6. Znamenskaya I.A., Ivanov I.E., Orlov D.M., Sysoev N.N. Pulsed action on a shock wave in the case of self-localized high-current surface discharge in front of the shock-wave front. // Doklady Physics, 2009. V. 54. № 3. P. 107-110. DOI: 10.1134/S102833580903001X

7. Ju Y. and Sun W. Plasma assisted combustion: Dynamics and chemistry. Prog. Energy Combust. Sci. 2015. V. 48, P.21-83. https://doi.org/10.1016/j.pecs.2014.12.002

8. Correale G., Michelis T., Ragni D., Kotsonis M. and Scarano F. Nanosecond-pulsed plasma actuation in quiescent air and laminar boundary layer. J. Phys. D: Appl. Phys., 2014. V. 47, 105201.

9. Raizer Yu. P. Gas Discharge Physics (Springer, Berlin, 1991)

10. Borovoy V.Y., Mosharov V.E., Radchenko V.N., Skuratov A.S., Struminskaya I.V. Leading edge bluntness effect on the flow in a model air-inlet. // Fluid Dynamics. 2014. V. 49. № 4. P. 454-467. https://doi.org/10.1134/So015462814040061 
11. Hadjadj A., Perrot Y., Verma S. Numerical study of shock/boundary layer interaction in supersonic overexpanded nozzles. // Aerosp. Sci. Technol. 2015. V. 42. P. 158-168. DOI: 10.1016/j.ast.2015.01.010

12. Georgievskii P. Y., Levin V. A. Control of the flow past bodies using localized energy addition to the supersonic oncoming flow. // Fluid Dynamics, 2003. Vol. 38, № 5 . P. 794-805. https://doi.org/10.1023/B:FLUI.0000007841.91654.10

13. Mursenkova I.V., Sazonov A.S., and Yu. Liao. The effect of pulsed sliding surface discharges on supersonic airflow past a thin wedge in shock tube. // Technical Physics Letters, 2018 V. 44. No. 2. P. 157-159. DOI: 10.1134/S1063785018020256

14. Mursenkova I.V., Liao Yu., Ivanov I.E., and Sysoev N.N. The characteristics of a nanosecond surface sliding discharge in a supersonic airflow flowing around a thin wedge. // Moscow University Physics Bulletin, 2019. V. 74, No. 3. P. 269-276. https://doi.org/10.3103/Soo27134919030093

15. Mursenkova I., Sazonov A., Liao Yu., Ivanov I. Visualization of the interaction region of an oblique shock wave with a boundary layer by the radiation of a nanosecond surface sliding discharge. // Scientific Visualization, 2019. V. 11, No 3, p. 76-87. DOI: 10.26583/sv.11.3.07

16. Ya.B. Zel'dovich and Yu.P. Raizer. Physics of Shock Waves and High-Temperature Hydrodynamic Phenomena. (Academic Press, New York, 2002). 